PREPARED FOR THE U.S. DEPARTMENT OF ENERGY, UNDER CONTRACT DE-AC02-76CH03073

PPPL-3483

PPPL-3483

UC-70

Stabilization of Tokamak Plasma by Lithium Streams

by

L.E. Zakharov

August 2000

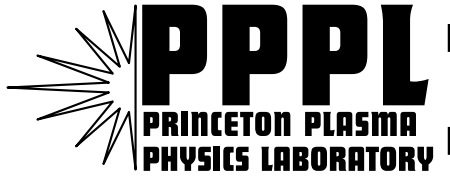

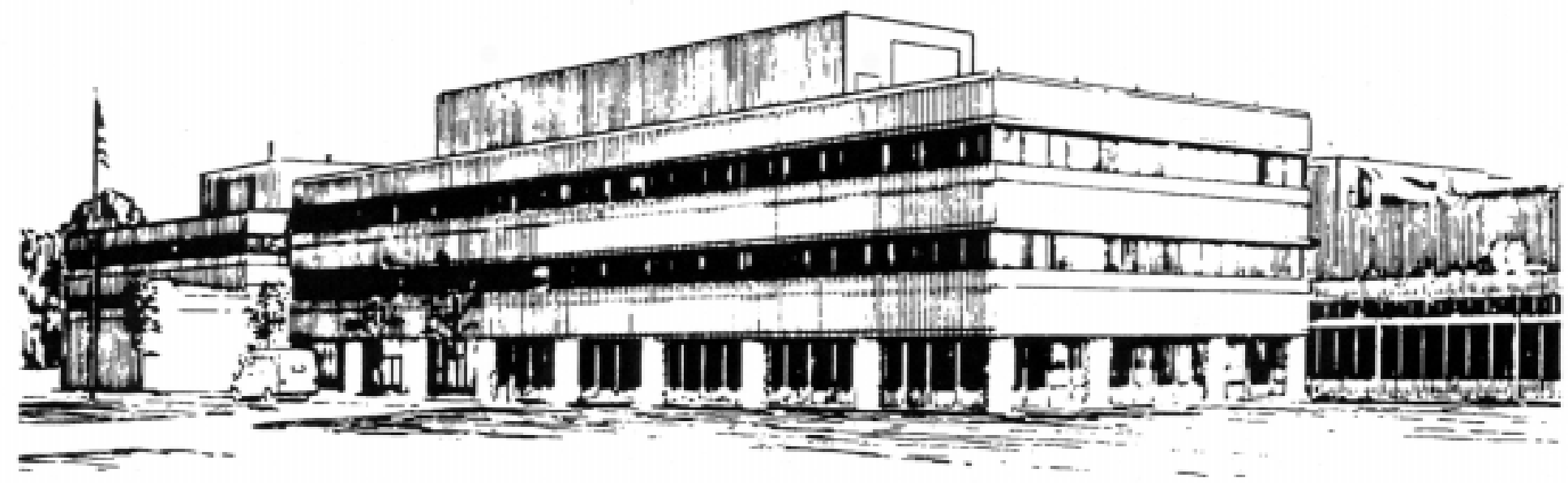

PRINCETON PLASMA PHYSICS LABORATORY PRINCETON UNIVERSITY, PRINCETON, NEW JERSEY 


\section{PPPL Reports Disclaimer}

This report was prepared as an account of work sponsored by an agency of the United States Government. Neither the United States Government nor any agency thereof, nor any of their employees, makes any warranty, express or implied, or assumes any legal liability or responsibility for the accuracy, completeness, or usefulness of any information, apparatus, product, or process disclosed, or represents that its use would not infringe privately owned rights. Reference herein to any specific commercial product, process, or service by trade name, trademark, manufacturer, or otherwise, does not necessarily constitute or imply its endorsement, recommendation, or favoring by the United States Government or any agency thereof. The views and opinions of authors expressed herein do not necessarily state or reflect those of the United States Government or any agency thereof.

\section{Availability}

This report is posted on the U.S. Department of Energy's Princeton Plasma Physics Laboratory Publications and Reports web site in Calendar Year 2000. The home page for PPPL Reports and Publications is: http://www.pppl.gov/pub_report/

DOE and DOE Contractors can obtain copies of this report from:

U.S. Department of Energy

Office of Scientific and Technical Information

DOE Technical Information Services (DTIS)

P.O. Box 62

Oak Ridge, TN 37831

Telephone: (865) 576-8401

Fax: (865) 576-5728

Email: reports@adonis.osti.gov

This report is available to the general public from:

National Technical Information Service

U.S. Department of Commerce

5285 Port Royal Road

Springfield, VA 22161

Telephone: $1-800-553-6847$ or

(703) $605-6000$

Fax: (703) 321-8547

Internet: http://www.ntis.gov/ordering.htm 


\title{
Stabilization of tokamak plasma by lithium streams
}

\author{
L. E. Zakharov, \\ Princeton Plasma Physics Laboratory, Princeton, NJ 08543-0451
}

June 8, 2000

\begin{abstract}
The stabilization theory of free-boundary magnetohydrodynamic instabilities in tokamaks by liquid lithium streams driven by magnetic propulsion is formulated. While the conventional, wall-locked, resistive wall mode can be well suppressed by the flow, a new, stream-locked mode determines the limits of the flow stabilization.
\end{abstract}

\section{Introduction}

Recently, the author proposed a mechanism of magnetic propulsion for driving fast lithium streams along the plasma facing surface of the tokamak vacuum chamber (Fig.1a). The metal is maintained at the first-wall surface by the electromagnetic force $\mathbf{J} \times \mathbf{B}$ (Fig.1b), where $\mathbf{J}$ is the poloidal current, driven through the liquid metal by an external power source and $\mathbf{B}$ is the tokamak magnetic field. The magnetic pressure, created in the fluid, is nonuniform along the lithium layer and the fluid is propelled from the high-field side of the tokamak to the low-field side in the form of two streams along the top and bottom halves of the vacuum chamber, respectively.

Because of the strong toroidal magnetic field, the $\mathbf{J} \times \mathbf{B}$ force can be made sufficient to create a pressure to the wall exceeding the external pressure, e.g., $1 \mathrm{~atm}$. This enables expulsion of the lithium from the vacuum chamber. Accordingly, the pressure drop along the streams exceeds $1-2 \mathrm{~atm}$, thus, making velocities of the order of $20 \mathrm{~m} / \mathrm{sec}$ typical for the lithium streams.

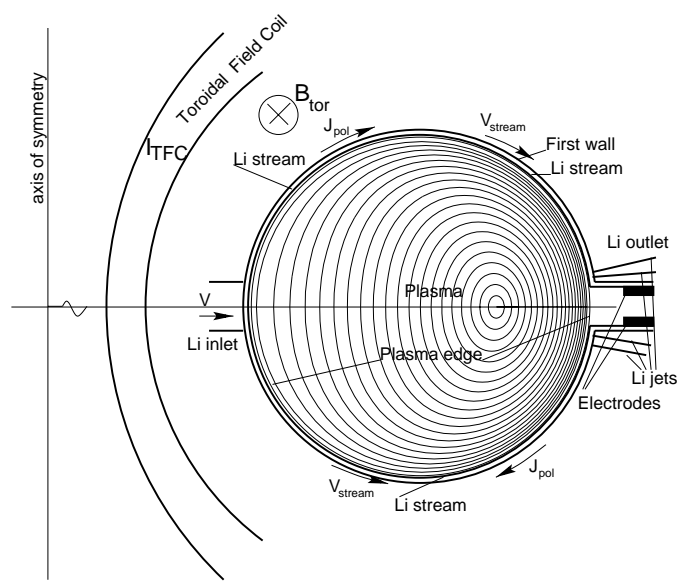

(a)

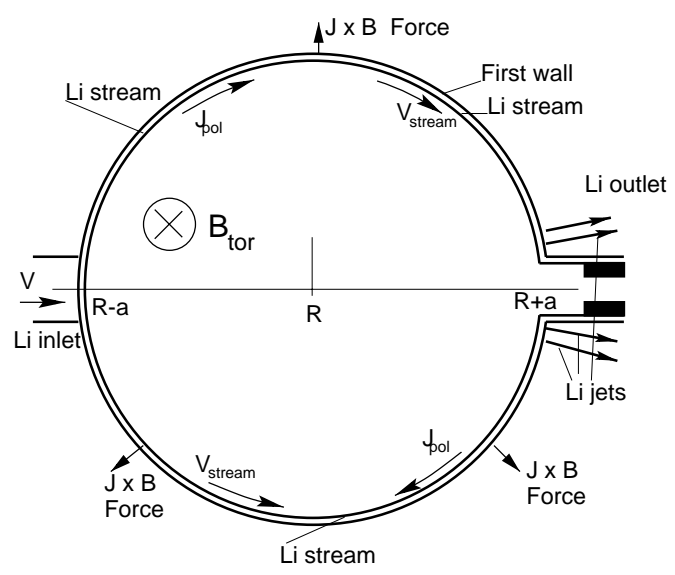

(b)

Fig.1. (a) Cross section of the tokamak with lithium streams (inlet and outlet are both schematic). (b) Guide wall with two lithium streams at the top and bottom halves of the chamber. 
These intense lithium streams have been proposed primarily as a new power extraction scheme compatible with a fusion reactor environment. Remarkably, due to centrifugal force, the streams themselves are robustly stable with respect to the sausage instability, associated with the electric current in the metal as well as to the much weaker gravitational instability. The big, externally controlled magnetic pressure due to the $\mathbf{J} \times \mathbf{B}$ force, holding streams on the wall, makes liquid metal insensitive to other electromagnetic forces even in the most violent disruption event in tokamaks.

In this paper, it is shown that intense lithium streams improve stability of the free-boundary modes of the tokamak plasma. In particular, lithium streams can potentially stabilize configurations which are otherwise unstable in the presence of a passive walls.

There are two stabilizing effects associated with lithium streams. First, streams enable an effective conducting shell right at the plasma boundary, thus, increasing significantly the effectiveness of a possible feedback stabilization. Second, the axisymmetric $m=1, n=0(m, n$ are the poloidal and toroidal wave numbers, respectively) pattern of the fast metal flow affects explicitly the resistive wall modes[1, 2$]$.

The first effect is straightforward and analogous to the theory of resistive (solid) wall modes, which have been extensively analyzed in the literature (see, e.g., References $[1,2,3,4,5,6,7,8,9]$ ) and where the authors tried to rely on nonideal effects in the plasma. The second effect, considered in this paper, has no direct analog in to existing theories. It provides a stabilization mechanism independent of the plasma properties.

\section{Interaction of lithium streams with external plasma modes}

If a plasma column is surrounded by a conducting shell (with or without metal flow) and if the plasma is stable with respect to internal (fixed boundary) MHD modes, then the remaining instabilities are the free boundary, so-called "resistive wall modes" $(\mathrm{RWM})[1,2]$, which are purely growing modes in the laboratory frame. Their growth rate $\gamma$ is determined by the resistive penetration time $\tau_{\text {res }}$

$$
\gamma \simeq \frac{1}{\tau_{\text {res }}}, \quad \tau_{\text {res }} \equiv \mu_{0} \sigma a h,
$$

where $\sigma$ is the conductivity of the wall, $a$ a characteristic minor radius, and $h$ the height of the metal shell (we dropped the poloidal wave number from the definition of $\tau_{r e s}$ ). With such a small growth rate, the plasma inertia can be neglected and the equations of motion for the plasma are those of perturbed equilibria. As a result, the growth rate is determined solely by the time evolution of the currents in the shell.

The metal flow and associated $\mathbf{V} \times \mathbf{B}$ electromotive force ( $\mathbf{V}$ is the flow velocity) affect the excitation and evolution of eddy currents and, thus, also stability. The stabilization depends significantly on the flow pattern. Thus, just a rotating metal shell is not effective because the RWM can be locked into rotation[2]. On the other hand, the two stream pattern of magnetic propulsion (Fig.1.), when the metal is supplied from the large toroidal field side of the tokamak and taken out from the low field side, eliminates the possibility of good mode locking. Instead, the mode can be locked only into one of the streams, thus, making the other stream equivalent to a perfectly conducting shell.

This physics consideration can be formalized and linked to the existing stability theory. Perturbations in the magnetic configuration can be expressed in terms of the perturbations of the vector potential

$$
\mathbf{A}=\tilde{\mathbf{A}} e^{\gamma t},
$$

where $\gamma$ is the linear (complex) growth rate. We will use a toroidal flux coordinate system $\rho, \theta, \varphi$ corresponding to the straight field-line representation of magnetic field with axisymmetric azimuth $\varphi$ and with poloidal angle $\theta=0$ on the high-field side. Inside the plasma, $\rho=$ const surfaces coincide with magnetic surfaces. The inner (plasma facing) and outer surface of the metal shell are specified by equations $\rho=a$ and $\rho=a+\delta a$ $(\delta a \ll a)$. Outside the shell the coordinates represent a continuous extension of internal coordinates with, e.g., $\rho=\infty$ corresponding to infinity and to the axis of symmetry. 
Vector potential can be taken in the following covariant form

$$
\tilde{\mathbf{A}} \equiv \eta(\rho, \theta, \varphi) \nabla \rho+\phi(\rho, \varphi) \nabla \theta+\psi(\rho, \theta, \varphi) \nabla \varphi, \quad \oint \psi d \theta=0,
$$

where $\phi$ does not depend on $\theta$ and $\psi$ is an oscillatory function of $\theta$. The perturbation of the magnetic field can be expressed either in contravariant form

$$
\tilde{\mathbf{B}}=\left(\psi_{\theta}^{\prime}-\phi_{\varphi}^{\prime}\right)(\nabla \theta \times \nabla \varphi)+\left(\eta_{\varphi}^{\prime}-\psi_{\rho}^{\prime}\right)(\nabla \varphi \times \nabla \rho)+\left(\phi_{\rho}^{\prime}-\eta_{\theta}^{\prime}\right)(\nabla \rho \times \nabla \theta)
$$

or in covariant form

$$
\tilde{\mathbf{B}}=\tilde{B}_{\rho} \nabla \rho+\tilde{B}_{\theta} \nabla \theta+\tilde{B}_{\varphi} \nabla \varphi .
$$

These two representations are related through the metric tensor $g_{i k}$ of the coordinate system

$$
\tilde{B}_{i}=\sum_{k} g_{i k} \tilde{B}^{k}, \quad i=\rho, \theta, \varphi, \quad k=\rho, \theta, \varphi,
$$

thus, making it possible to express the covariant components $\tilde{B}_{\theta, \theta, \varphi}$ in terms of $\eta, \phi, \psi$. Both inside and outside the plasma, the equations for the unknowns $\eta, \phi, \psi$ can be written in the form of contravariant components of Ampere's law $(\nabla \times \tilde{\mathbf{B}})=\mu_{0} \mathbf{j}$

$$
J(\mathbf{j} \cdot \nabla \rho)=\left(\tilde{B}_{\varphi}\right)_{\theta}^{\prime}-\left(\tilde{B}_{\theta}\right)_{\varphi}^{\prime}, \quad J(\mathbf{j} \cdot \nabla \theta)=\left(\tilde{B}_{\rho}\right)_{\varphi}^{\prime}-\left(\tilde{B}_{\varphi}\right)_{\rho}^{\prime} \quad \text { and } \quad J(\mathbf{j} \cdot \nabla \varphi)=\left(\tilde{B}_{\theta}\right)_{\rho}^{\prime}-\left(\tilde{B}_{\rho}\right)_{\theta}^{\prime},
$$

where $J$ is the Jacobian of the coordinate system

$$
J \equiv \frac{D(x, y, z)}{D(\rho, \theta, \varphi)} .
$$

Because $\nabla \cdot \mathbf{j}=0$, not all of the three equations in (2.7) are independent. Only the first equation together with the $\theta$-averaged component of the second equation and the oscillatory part of the third equation are essential. The boundary conditions for these equations are:

$$
\left.\tilde{\mathbf{A}}\right|_{\rho=0}=\left.\tilde{\mathbf{A}}\right|_{\rho=\infty}=0 .
$$

Inside the plasma, the expressions for the current density can be obtained from the perturbed equilibrium equations. In the vacuum region $\mathbf{j}=0$ while inside the flowing metal, $\mathbf{j}$ is determined by Ohm's law.

We consider two lithium streams flowing along the plasma boundary from the low-field side of the torus to the high-field side. The velocity of the flow is assumed to be purely poloidal, $\mathbf{V}=V(\theta) \mathbf{e}_{p o l}$, where $\mathbf{e}_{p o l}$ is the unit vector in the poloidal direction. For each stream, the velocity $V(\theta)$ and the height $h(\theta)$ of the stream are related by equations

$$
\left.V(\theta)\right|_{0<\theta<\pi}=V \frac{R h}{r_{w}(\theta) h(\theta)},\left.\quad V(\theta)\right|_{-\pi<\theta<0}=-V \frac{R h}{r_{w}(\theta) h(\theta)},
$$

where $r=r_{w}(\theta)$ together with $z=z_{w}(\theta)$ is a parametric representation of the wall contour, $R$ is a characteristic major radius, and $V$ and $h$ are the velocity and height of the flow at this radius, respectively.

The current density in the thin shell can be written in terms of the stream function $I(\theta, \varphi)$ :

$$
\mu_{0} \tilde{\mathbf{j}}_{w} \equiv \frac{\nabla \rho \times \nabla I}{\delta a}=-\frac{I_{\varphi}^{\prime}}{J_{w}} \mathbf{e}_{\theta}+\frac{I_{\theta}^{\prime}}{J_{w}} \mathbf{e}_{\varphi}, \quad \mathbf{e}_{\varphi} \equiv r \mathbf{e}_{t o r}, \quad\left|\mathbf{e}_{t o r}\right|=1 .
$$

Here, $J_{w}$ plays the role of the Jacobian for an infinitesimally thin shell

$$
J_{w} \equiv r_{w}(\theta) h(\theta) \sqrt{g_{\theta \theta}}, \quad g_{\theta \theta}=\left(r_{w}\right)_{\theta}^{\prime 2}+\left(z_{w}\right)_{\theta}^{\prime 2} \quad \text { and } \quad\left|\mathbf{e}_{\theta}\right|=\sqrt{g_{\theta \theta}} .
$$


With this representation the matching conditions across the shell can be written as:

$$
\left(\left.\tilde{B}_{\varphi}\right|_{a+\delta a}-\left.\tilde{B}_{\varphi}\right|_{a}\right)_{0}=\left(I_{\varphi}^{\prime}\right)_{0},\left.\quad \tilde{B}_{\theta}\right|_{a+\delta a}-\left.\tilde{B}_{\theta}\right|_{a}=I_{\theta}^{\prime},
$$

where $(\ldots)_{0}$ means averaging over $\theta$. In Ohm'law

$$
\tilde{\mathbf{j}}_{w}=\sigma\left[-\gamma \tilde{\mathbf{A}}-\nabla \phi_{E}+(\mathbf{V} \times \tilde{\mathbf{B}})\right]
$$

two essential (covariant) components have the form

$$
-\frac{g_{\theta \theta}}{J_{w} \mu_{0} \sigma} I_{\varphi}^{\prime}=-\gamma \phi-\left(\phi_{E}\right)_{\theta}^{\prime}, \quad \frac{r^{2}}{J_{w} \mu_{0} \sigma} I_{\theta}^{\prime}=-\gamma \psi-\left(\phi_{E}\right)_{\varphi}^{\prime}-V^{\theta}\left(\psi_{\theta}^{\prime}-\phi_{\varphi}^{\prime}\right) .
$$

After elimination of the scalar potential $\phi_{E}$, an equation for the stream function $I$ is given:

$$
\frac{h g_{\theta \theta}}{J_{w}} I_{\varphi \varphi}^{\prime \prime}+\left(\frac{h r^{2}}{J_{w}} I_{\theta}^{\prime}\right)_{\theta}^{\prime}=-\mu_{0} h \sigma \gamma\left(\psi_{\theta}^{\prime}-\phi_{\varphi}^{\prime}\right)-R_{m}\left[\frac{v(\theta)\left(\psi_{\theta}^{\prime}-\phi_{\varphi}^{\prime}\right)}{\sqrt{g_{\theta \theta}}}\right]_{\theta}^{\prime}, \quad \text { where } \quad v(\theta) \equiv \frac{V(\theta)}{V} .
$$

In Eq.(2.16), $R_{m}$ is the magnetic Reynolds number for the flow calculated based on the height of the stream

$$
R_{m} \equiv \mu_{0} \sigma V h .
$$

For lithium, $\mu_{0} \sigma \simeq 4 \mathrm{sec} / \mathrm{m}^{2}$. Thus, with characteristic velocities of the order of $20 \mathrm{~m} / \mathrm{sec}$, it is possible to obtain Reynolds numbers of the order of unity even for thin lithium streams $h \simeq 1 \mathrm{~cm}$.

Because of the gap in the wall at the outlet point, $I$ should satisfy boundary conditions

$$
I(\omega=\pi-\alpha, \varphi)=\text { const }, \quad I(\omega=\pi+\alpha, \varphi)=\text { const }
$$

at the gap sides $\omega=\pi \pm \alpha$, where the angle $2 \alpha$ specifies the extend of the gap.

Equations (2.7) with boundary conditions of Eq. (2.9), matching conditions across the shell (2.13) and Eq. (2.16) specify the eigenvalue problem for obtaining the growth rate $\gamma$.

Equations (2.7) are typically being solved using a Fourier transformation

$$
\begin{aligned}
& \eta=\sum_{m} \eta_{m}(\rho) e^{i m \theta-i n \varphi}, \quad \phi \equiv-\psi_{0}(\rho) e^{-i n \varphi}, \quad \psi=\sum_{m \neq 0} \psi_{m}(\rho) e^{i m \theta-i n \varphi}, \\
& (\vec{\eta})_{m} \equiv \eta_{m} \quad \text { and } \quad(\vec{\psi})_{m} \equiv \psi_{m},
\end{aligned}
$$

where two vector functions $\vec{\eta}$ and $\vec{\psi}$ are composed from the Fourier harmonics of the components of the vector potential. Both inside the plasma and in the vacuum region, the first equation in (2.7) allows to express vector $\vec{\eta}$ in terms of $\vec{\psi}^{\prime}$ and $\vec{\psi}$. Thus, the full set of independent solutions to Eqs.(2.7), satisfying boundary conditions (2.9), can be represented in the form of the superposition of the normalized fundamental solutions to Euler's equations

$$
\vec{\psi}_{p}(\rho)=\bigcup_{p}(\rho) \vec{\psi}(a), \quad \vec{\psi}_{v}(\rho)=\bigcup_{v}(\rho) \vec{\psi}(a), \quad \bigcup_{p}(0)=0, \quad \bigcup_{v}(\infty)=0 \quad \text { and } \quad \bigcup_{p, v}(a)=\mathrm{I},
$$

where each column $\bigcup_{p, v}^{m^{\prime}}$ in matrix functions $\bigcup_{p, v}$ represents an independent solution to Eqs.(2.7) either in the plasma (index " $p$ ") or in the vacuum (index " $v$ ") regions. At the plasma boundary $\rho=a$, the $\bigcup_{p, v}$ are the identity matrices, $\mathrm{U}_{p, v}(a)=\mathrm{I}$.

In addition to the matrices $\bigcup_{p, v}$ of the fundamental solutions, it is possible to introduce related matrices $\mathrm{D}_{p, v}$, representing the left-hand side of the matching conditions (2.13) in Fourier space for every fundamental solution $\bigcup_{p, v}^{m^{\prime}}$

$$
\begin{aligned}
& \tilde{B}_{\varphi}(a, \theta, \varphi)=\sum_{m \neq 0} \tilde{B}_{\theta, m} e^{i m \theta-i n \varphi}, \quad \tilde{B}_{\varphi}(a, \theta, \varphi)=\sum_{m} \tilde{B}_{\varphi, m} e^{i m \theta-i n \varphi} \\
& (\vec{D})_{m \neq 0, m^{\prime}} \equiv-\tilde{B}_{\theta, m \neq 0}^{m^{\prime}} \quad \text { and } \quad(\vec{D})_{0, m^{\prime}} \equiv-\tilde{B}_{\varphi, 0}^{m^{\prime}}
\end{aligned}
$$


Using the matrices $D_{p, v}$, the matching conditions (2.13) can be written in the form

$$
\left(\mathrm{D}_{v}-\mathrm{D}_{p}\right) \vec{\psi}(a)=-i \mathrm{M} \vec{I}
$$

where vector $\vec{I}$ and matrix $\mathrm{M}$ are defined as

$$
I(a, \theta, \varphi) \equiv \sum_{m}(\vec{I})_{m} e^{i m \theta-i n \varphi}, \quad \mathrm{M}_{m \neq 0, m^{\prime}} \equiv m \delta_{m m^{\prime}} \quad \text { and } \quad \mathrm{M}_{0, m^{\prime}} \equiv-n \delta_{0 m^{\prime}} .
$$

Eq. (2.16) for $I$ can be written in the matrix form as

$$
\mathrm{S} \vec{I}=i \mu_{0} h \sigma \gamma \mathrm{M} \vec{\psi}-R_{m} \mathrm{VM} \vec{\psi}
$$

where the matrices $\mathrm{S}$ and $\mathrm{V}$ are defined as

$$
(\mathrm{S})_{m m^{\prime}}=m m^{\prime}\left(h \frac{r^{2}}{J_{w}}\right)_{m-m^{\prime}}+n^{2}\left(\frac{h g_{\theta \theta}}{J_{w}}\right)_{m-m^{\prime}} \quad \text { and } \quad(\mathrm{V})_{m m^{\prime}} \equiv m\left(\frac{v(\theta)}{\sqrt{g_{\theta \theta}}}\right)_{m-m^{\prime}}
$$

Its solution, expressed in the form

$$
\vec{I}=i \mu_{0} h \sigma \gamma \mathrm{S}^{-1} \mathrm{M} \vec{\psi}-R_{m} \mathrm{~S}^{-1} \mathrm{VM} \vec{\psi},
$$

after subsitution into matching conditions (2.22) gives a dispersion relation

$$
\left(\mathrm{D}_{v}-\mathrm{D}_{p}\right) \vec{\psi}(a)=\mu_{0} h \sigma \gamma\left(\mathrm{MS}^{-1} \mathrm{M}\right) \vec{\psi}+i R_{m}\left(\mathrm{MS}^{-1} \mathrm{VM}\right) \vec{\psi}
$$

For any plasma equilibrium, $\mathrm{D}_{v}, \mathrm{D}_{p}$ can be calculated using standard stability codes for free-boundary MHD modes. Then, the growth rates and stability conditions in presence of flow can be obtained by solving the linear algebraic eigenvalue problem (2.27) for $\gamma$.

The final Eq. (2.27) of the present theory has the following structure. The matrices $D_{v}-D_{p}$ and $M S^{-1} M$ (which are not related to flow) are Hermitian. Their off-diagonal terms containing, in particular, the toroidal ballooning mode coupling, make a destabilizing contribution. The matrix $i R_{m} \mathrm{MS}^{-1} \mathrm{VM}$ describing the flow effect is essentially anti-Hermitian. Its off-diagonal terms, producing the mode coupling, contribute positively to stability.

\section{Stabilization of current-driven kink modes}

In order to illustrate the stabilizing effect of the streams, we consider a simple model of a tokamak plasma with a circular cross section in the large aspect ratio approximation corresponding to $a / R \ll 1, B_{\text {pol }} / B_{\text {tor }} \ll 1$ and neglect pressure effect. For simplicity, we also neglect the effect of a gap in the flow near the outlet point and assume that $I$ is a periodic function of $\omega, \varphi$.

In this case Eqs.(2.7) in the plasma and vacuum regions are reduced to a second-order equation

$$
\left(\rho \psi_{m}^{\prime}\right)^{\prime}=\frac{m^{2}}{\rho} \psi+\frac{\mu_{0} R j^{\prime}}{B_{t o r}(\mu-n / m)} \psi, \quad \mu(\rho) \equiv \frac{\mu_{0} R}{B_{t o r} \rho} \int_{0}^{\rho} j(\rho) \rho d \rho, \quad \mu(\rho)=\frac{1}{q},
$$

where $j(\rho)$ is the toroidal current distribution and $q$ is the safety factor. Matrices $\mathbf{D}_{p}, \mathbf{D}_{v}, \mathbf{S}$ are diagonal:

$$
\left(\mathrm{D}_{p}\right)_{m m^{\prime}}=\frac{a \psi_{p, m}^{\prime}}{R \psi_{m}} \delta_{m m^{\prime}}, \quad\left(\mathrm{D}_{v}\right)_{m m^{\prime}}=\frac{a \psi_{v, m}^{\prime}}{R \psi_{m}} \delta_{m m^{\prime}}=-\frac{m}{R} \delta_{m m^{\prime}} \quad \text { and } \quad(\mathrm{S})_{m m^{\prime}}=m^{2} \frac{R}{a} \delta_{m m^{\prime}} .
$$


For an example of a constant velocity of each stream, the normalized stream velocity $v(\theta)$ in Eq.(2.16) has the following Fourier expansion

$$
\left.v(\theta)\right|_{0<\theta<\pi}=1,\left.\quad v(\omega)\right|_{-\pi<\theta<0}=-1, \quad v(\theta)=\sum_{k} v_{2 k+1} e^{i(2 k+1) \theta}, \quad v_{2 k+1}=\frac{2}{i \pi(2 k+1)},
$$

and the flow coupling matrix $\mathrm{V}$ can be calculated as

$$
(\mathrm{V})_{m, m+2 k+1}=\frac{m}{a} v_{-2 k-1}=\frac{m}{a} v_{2 k+1}^{*}, \quad(\mathrm{~V})_{m, m+2 k}=0
$$

The dispersion relation can be written as an eigenvalue problem for a system of linear equations

$$
a \Delta_{m}^{\prime} \psi_{m}=\tau_{r e s} \gamma \psi_{m}+R_{m} \sum_{k}(m+2 k+1) v_{2 k+1}^{*} \psi_{m+2 k+1}, \quad \tau_{r e s}=\mu_{0} \sigma h a,
$$

where

$$
a \Delta_{m}^{\prime} \equiv R\left(\mathbf{D}_{v}-\mathbf{D}_{p}\right)_{m m}=\left.a \frac{\psi_{v, m}^{\prime}-\psi_{p, m}^{\prime}}{\psi_{m}}\right|_{\rho=a} .
$$

For a classical example[10] of a plasma with uniform current distribution, $a \Delta_{m}^{\prime}$ has an analytical representation

$$
a \Delta_{m}^{\prime}=-2 m \frac{m-1-n q}{m-n q}, \quad q(\rho)=\mathrm{const}=q_{a} .
$$

In order to make stabilization by the flow evident, let us consider a three harmonics approximation for $\psi$

$$
\psi=\left(\psi_{m-1} e^{i(m-1) \theta}+\psi_{m} e^{i m \theta}+\psi_{m+1} e^{i(m+1) \theta}\right) e^{-i n \varphi+\gamma t}
$$

In this case, the eigenvalue problem for linear system

$$
\begin{aligned}
& \left(a \Delta_{m-1}^{\prime}-\tau_{r e s} \gamma\right) \psi_{m-1}=i m R_{m} v_{1}^{*} \psi_{m} \\
& \left(a \Delta_{m}^{\prime}-\tau_{r e s} \gamma\right) \psi_{m}=i(m+1) R_{m} v_{1}^{*} \psi_{m+1}+i(m-1) R_{m} v_{1} \psi_{m-1} \\
& \left(a \Delta_{m+1}^{\prime}-\tau_{r e s} \gamma\right) \psi_{m+1}=i m R_{m} v_{1} \psi_{m}
\end{aligned}
$$

is reduced to solving an algebraic equation for $\gamma$ :

$$
a \Delta_{m}^{\prime}-\tau_{r e s} \gamma+\frac{m(m+1) R_{m}^{2}\left|v_{1}\right|^{2}}{a \Delta_{m+1}^{\prime}-\tau_{r e s} \gamma}+\frac{m(m-1) R_{m}^{2}\left|v_{1}\right|^{2}}{a \Delta_{m-1}^{\prime}-\tau_{r e s} \gamma}=0 .
$$

In a typical situation of an unstable low $m, n$ mode with $a \Delta_{m}^{\prime}>0$, the satellite modes are stable, i.e., $a \Delta_{m-1}^{\prime}<0$ and $a \Delta_{m+1}^{\prime}<0$. Taking this into account, the above equation shows explicitly that the flow has a stabilizing effect (proportional to $R_{m}^{2}$ ) on the mode with the real growth rate.

Growth rates calculated for the $m=3$ current-driven kink mode and uniform current distribution are shown in Fig. 2a. 

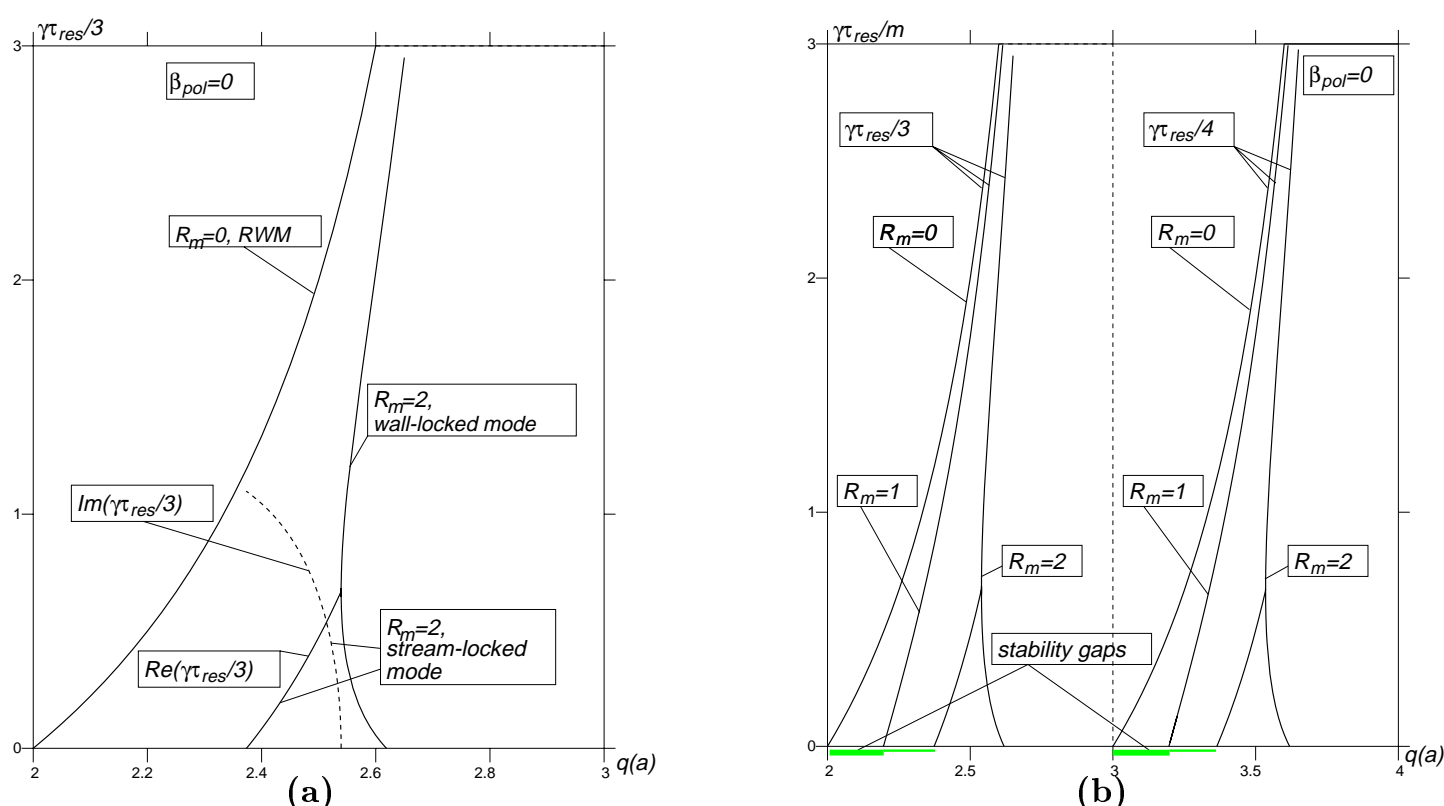

Fig. 2. Normalized growth rate (solid curves) and rotation frequency (dashed curves) of RWM for a circular plasma with a uniform current distribution and $\beta_{p o l}=0$. (a) wall- and stream-locked $m=3$ mode; (b) stability diagram for $2<q_{a}<4$.

In the absence of flow, the $m=3$ mode is robustly unstable[1,2] over the entire region $2 \leq q_{a} \leq 3$. With no resonant surfaces inside the plasma, this configuration cannot be stabilized by the nonideal plasma effects considered in the literature $[3,4,5,6,7,8,9]$. The metal flow creates a stability gap $2 \leq q_{a} \leq 2.37$.

Fig. 2a shows, that in addition to the conventional wall-locked mode, which has a purely real growth rate $\Im(\gamma)=0$, Eq. (3.10) contains additional roots corresponding to a new mode which rotates and is locked into one of the streams, $\Im(\gamma) \neq 0$. For this mode, another stream acts as a perfectly conducting wall. While the wall-locked mode is well affected by the velocity of the streams, the stream-locked mode is insensitive to it and determines the limits for the flow stabilization.

\section{Stabilization of the pressure-driven kink modes}

Plasma pressure introduces an additional destabilization of the tokamak equilibrium configuration. In the simplest case of the plasma with a circular cross section and low, but finite, pressure $p(\rho)$ it is described by an energy principle

$$
\begin{aligned}
W= & \sum_{m} \frac{\pi}{R} \int\left\{\rho\left|\psi_{m}^{\prime}\right|^{2}+\frac{m^{2}}{\rho}\left|\psi_{m}\right|^{2}+\frac{j^{\prime} R}{B\left(\mu-\mu_{m}\right)}\left|\psi_{m}\right|^{2}+4 \frac{\rho}{R^{2}} \beta_{p o l} m^{2} \frac{2 \beta_{p o l}+1-\frac{n^{2}}{m^{2}}}{(m-n q)^{2}}\left|\psi_{m}\right|^{2}\right. \\
& -2 \frac{\beta_{p o l}}{R} \frac{m(m+1) \psi_{m} \psi_{m+1}^{*}}{(m-n q)(m+1-n q)}-2 \frac{\beta_{p o l}}{R} \frac{m(m-1) \psi_{m} \psi_{m-1}^{*}}{(m-n q)(m-1-n q)} \\
& \left.-2 \frac{\beta_{p o l}}{R} \frac{m \psi_{m}\left(\rho \psi_{m+1}^{\prime *}+\rho \psi_{m-1}^{\prime *}\right)}{m-n q}-2 \frac{\beta_{p o l}}{R} \frac{(m+1) \rho \psi_{m}^{\prime} \psi_{m+1}^{*}}{m+1-n q}-2 \frac{\beta_{p o l}}{R} \frac{(m-1) \rho \psi_{m}^{\prime} \psi_{m-1}^{*}}{m-1-n q}\right\} d \rho,
\end{aligned}
$$

which approximates[11, 12] the functional of potential energy. Here, the parameter $\beta_{\text {pol }}$ is defined as:

$$
\beta_{p o l}(\rho) \equiv-\frac{\mu_{0} \rho p^{\prime}(\rho)}{2 B_{p o l}^{2}(\rho)}
$$


and characterizes the local poloidal $\beta$. For a uniform current distribution and parabolic pressure profile $\beta_{p o l}(\rho)=$ const the Hermitian (symmetric) matrix $a \Delta_{m m^{\prime}}^{\prime} \equiv R\left(\mathrm{D}_{v}-\mathrm{D}_{p}\right)_{m m^{\prime}}$ can be calculated as

$$
\begin{aligned}
a \Delta_{m, m}^{\prime} & =-2 m \frac{m-1-n q}{m-n q}-\frac{m+2}{m+1} \frac{a^{2}}{R^{2}} \beta_{p o l}\left(\beta_{p o l}+1-\frac{n^{2}}{m^{2}}\right) \frac{m^{2}}{(m-n q)^{2}}, \\
a \Delta_{m-1, m}^{\prime} & =2 \frac{a}{R} \beta_{p o l} \frac{m-1}{m-1-n q}, \quad a \Delta_{m+1, m}^{\prime}=2 \frac{a}{R} \beta_{p o l} \frac{m}{m-n q} .
\end{aligned}
$$

Its off-diagonal terms lead to a toroidal mode coupling, which results in destabilization of free-boundary modes[12]. For a uniform current distribution, the RWM acquires a finite growth rate for all $q(a)$, as seen in Fig. 3. The metal streams improve the stability of the finite-beta plasma in a similar way as they affect the pure currrent-driven modes. Fig. 3a shows growth rates for both wall- and stream-locked modes for $a / R=1 / 4$ and $\beta_{p o l}=0.5$.

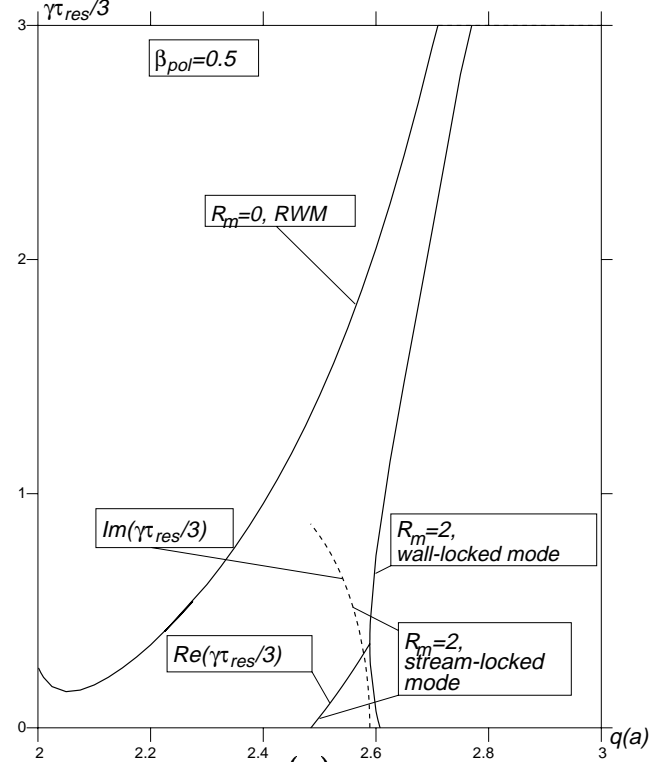

(a)

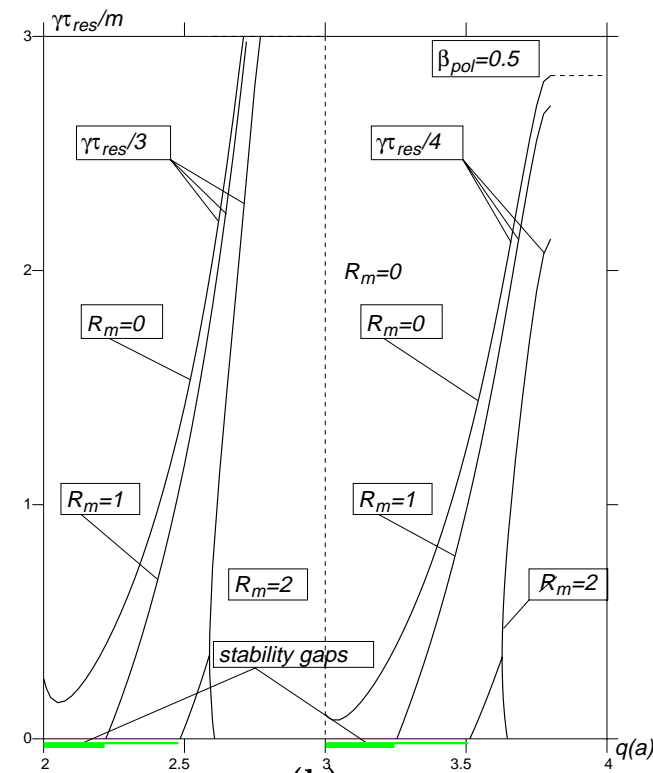

(b)

Fig.3. Normalized growth rate (solid curves) and rotation frequency (dashed curves) of RWM for a circular plasma $R / a=4$ with a uniform current distribution and $\beta_{\text {pol }}=0.5$. a) wall- and stream-locked $m=3$ mode; b) stability diagram for $2<q_{a}<4$.

With the metal flow, stability gaps appear for the finite-beta plasma even for a uniform current distribution. It is possible also to notice that for a given $n$, the size of these gaps is not sensitive to the poloidal mode number $m$ for both current and pressure driven free-boundary modes. Thus, both low- and high- $m$ modes can be stabilized by the lithium flow.

\section{Summary}

Lithium streams, driven by magnetic propulsion, lead to improved stability of the tokamak free-boundary modes. The stabilizing effect depends on the flow Reynolds number and is essential when $R_{m}$ exceeds 1 . In contrast to the "rotating" wall, the $m=1$ pattern of the stream flow may lead to a real stabilization of the plasma. The theoretical formalism presented in this paper allows to calculate the eddy currents in the streams, as well as to use the existing numerical codes for evaluation of growth rates and stability conditions for resistive wall instabilities in the presence of flow. 


\section{References}

[1] P. H. Rutherford, Basic Physical Processes of Toroidal Fusion Plasmas (Proceedings of the Courses Workshop held at Varenna, 1985) (CEC, Brussels, 1986), Vol. 2, p. 531.

[2] L. E. Zakharov and S. V. Putvinskii, Sov. J. Plasma Phys. 13, 68 (1987).

[3] A. Bondeson and D. J. Ward, Phys. Rev. Lett. 72, 2709 (1994).

[4] J. M. Finn, Phys. Plasmas 2, 3782 (1995).

[5] R. Betti and J. P. Freidberg, Phys. Rev. Lett. 74, 2949 (1995).

[6] J. M. Finn and R. A. Gerwin, Phys. Plasmas 3, 2344 (1996).

[7] A. Bondeson and D. X. Xie, Phys. Plasmas 4, 2081 (1997).

[8] J. M. Finn and C. P. Sovenic, Phys. Plasmas 5, 461 (1998).

[9] A. Bondeson, C. G. Gimblett, and R. J. Hastie, Phys. Plasmas 6, 637 (1999).

[10] V. D. Shafranov, Sov. Phys. - Techn. Phys. 40, 241 (1970).

[11] L. E. Zakharov, Nucl. Fusion 18, 335 (1978).

[12] O. P. Pogutse and E. I. Yurchenko, Nucl. Fusion 18, 1629 (1978). 
The Princeton Plasma Physics Laboratory is operated by Princeton University under contract with the U.S. Department of Energy.

\author{
Information Services \\ Princeton Plasma Physics Laboratory \\ P.O. Box 451 \\ Princeton, NJ 08543
}

Phone: 609-243-2750

Fax: 609-243-2751

e-mail: pppl_info@pppl.gov

Internet Address: http://www.pppl.gov 